\title{
Testing procedures for measuring oral vibrotactile thresholds: III. Effects obtained using a nonclamping method
}

\author{
KAL M. TELAGE \\ Sensory Communication Laboratory, School of Allied Health, Ithaca College, Ithaca, New York 14850 \\ and \\ LINDA A. PETROSINO \\ Department of Speech Pathology and Audiology, Ithaca College, Ithaca, New York 14850
}

\begin{abstract}
Lingual vibrotactile thresholds were obtained from 20 randomly selected adult subjects utilizing a modified testing technique which does not clamp the lingual structure. Results across five frequencies showed a flat nonsummating response that was significantly different from previous results in which some degree of lingual clamping was procedurally employed. Findings were consistent over test-retest conditions and supported the view that lingual clamping may distort tissues so as to excite ventrally based frequency-responsive mechanoreceptors.
\end{abstract}

Continued research using lingual vibrotactile stimulation has been directed toward establishing a viable and reliable clinical tool. This work has been primarily of a methodological nature, focusing upon modifications and refinements in instrumentation and testing procedures. The major clinical interest has been to provide a means for assessing the normalcy of the lingual-tactile sensory feedback system in young children. This interest has been fostered by servomechanistic conceptions of the articulatory process in which haptic sensory mechanisms interplay with motor synergies in the maturation and subsequent development of speech sounds.

A number of recent modifications have attempted to facilitate the evaluative process. The most notable has been to introduce a programmable automated feature which basically tracks and records threshold sensitivity at and across frequency while simultaneously adding and subtracting attenuation in the manner of Békésy audiometry. Findings for adult subjects have been most encouraging and reflect greater consistency in locating and maintaining thresholds for selected frequencies.

One methodological area of continued concern to us has been the apparent necessity to clamp the tongue in the test position. This technique was used in the prototype apparatus developed by Fucci (1972) and in a modified clamp assembly by Fucci and Kelly (1972). It has been considered important to provide an adequate resting platform for the tongue while simultaneously compressing the lingual tissue against the free-surround disk above. Although extreme care is taken in clamping the tongue in a way that assists subjects in maintaining position and allows for easy withdrawal, the procedure includes some experimental disadvantages. The first and most apparent is a natural anxiety concerning compressing the anterior free margin of the tongue. Even with desensitization, some subjects persist in a tendency to withdraw the lingual blade from between the clamp assembly. In most cases it is an unconscious reaction which occurs very slowly during testing. A second disadvantage is that the clamping procedure is in itself time consuming and becomes fatiguing for the subject. Generally, the tongue must be rested and then reclamped between each threshold trial.

Of further interest is the nature of mechanoreceptors excited using clamping as compared to stimulating the tongue directly. The work of Telage and Fucci (1973, 1974a, 1974b, 1975) has consistently demonstrated a differential frequency response in the range 100 to $600 \mathrm{~Hz}$. These findings have contrasted with research reported by Verrillo (1966, 1968), who compared thresholds obtained from the palmar surface with those obtained on the dorsal lingual surface. Thresholds obtained on the palm showed a U-shaped function across frequency, while the lingual response was flat. Verrillo concluded that there are at least two different mechanoreceptor systems in cutaneous tissue. One of the systems mediates predominantly high-frequency vibrations (above $60 \mathrm{~Hz}$ ) and responds in a selective manner to frequency parameters; the other determines the psychophysical response at lower frequencies and is not frequency bound. It is important to note that Verrillo did not clamp the lingual structure.

Histologically, the palmar surface is known to be densely populated by Pacinian corpuscles end organ receptors known to respond selectively and optimally 
to frequency (Geldard, 1972). By contrast, the dorsal surface of the tongue is considered to be devoid of this type of receptor (Winkelman, 1960). However, Pacinian corpuscles are considered to be present in deeper, more ventral aspects of the tongue (Verrillo, 1966). One possible explanation of the current discrepancy in threshold findings might be attributed to the "clamping factor." That is, in clamping the tongue, is it possible to push up toward the dorsal surface and excite Pacinian receptors that would otherwise not be excited? If so, a flat threshold response above $60 \mathrm{~Hz}$ would be hypothesized, if a nonclamping procedure was employed.

The purpose of this research is twofold. First, it attempts to evaluate the consistency of lingual thresholds obtained using a nonclamping lingual procedure. Second, it tests the prediction that the response characteristics across frequency will be nonsummating throughout the range tested.

\section{METHOD}

\section{Procedure}

Stimulus control unit. Figure 1 presents a block diagram of the instrument package used in this study. The stimulus control unit is composed of Coulbourn solid state logic modules. These units generate pulsed vibratory signals which may be varied in frequency, intensity, and temporal characteristics. Three universal timers are programmed to control signal duration and duty cycle. The timers gate a selectable rise/fall module on and off. This continuously adjustable electronic switch was set to generate a rise/fall time of $100 \mathrm{msec}$. The signal from the rise/fall module was fed into a precision signal generator and an audio mixer amplifier. Stimulus intensities were varied in increments of $256^{1 / 2}$-dB steps by passing them through a programmable attenuator and an 8-bit binary up/down counter. The subject interface consisted of a pushbutton switch connected to a switch input module and an AND gate. By pressing or releasing the switch, attenuation was added or subtracted, permitting subjects to track threshold sensitivity across frequency. Pulsed signals from the stimulus control unit drive the mini electromagnetic vibrator that was the stimulus-producing aggregate of the system.

Vibrotactile stimulator. Figure 2 presents a schematic dia-

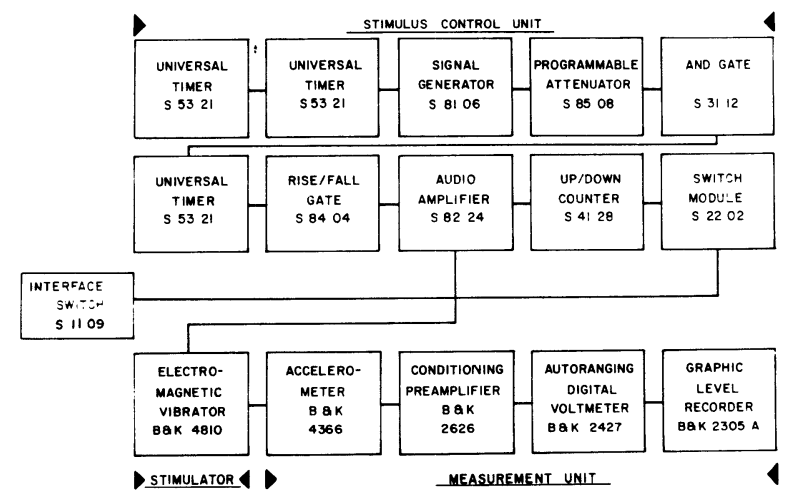

Figure 1. A block diagram of the automated instrumentation system.

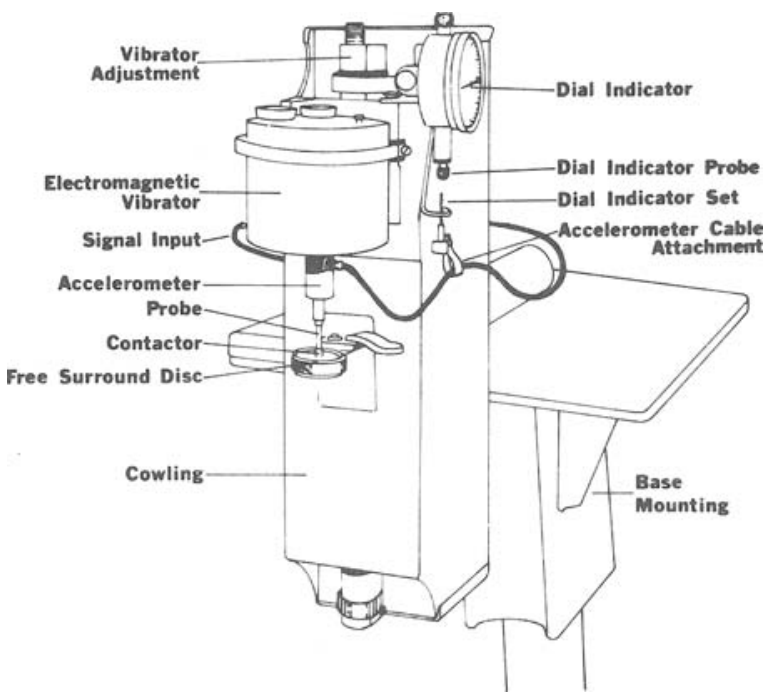

Figure 2. Schematic diagrams of the vibrator, free surround, and contactor adjustment aspects of the oral vibrotactile stimulator.

gram of the vibrator and modified clamp assembly portion of the vibrotactile stimulator. The lower clamping arm with disk was removed. The upper clamping arm with free-surround disk was retained. All contact points between the vibrator and the cowling are insulated by rubber stripping.

The vibrator probe with attached contactor was lowered through the upper free-surround disk until it established contact with the tongue. The area of the contactor was $.128 \mathrm{~cm}^{2}$. The diameter of the opening in the free surround was $2 \mathrm{~mm}$ larger than the diameter of the contactor.

Measurement unit. An accelerometer mounted on the probe measured contactor displacement as a small voltage. Displacement voltages were directly amplified by a conditioning preamplifier that was set to emit $100 \mathrm{mV} / \mathrm{G}$ of acceleration. Signals were constantly monitored by an autoranging digital volt meter set to read peak displacement values in millivolts.

\section{Subjects}

Twenty adult subjects ranging in age from 20 to 25 years were selected at random. None of the subjects reported a history of speech disorders or any known sensory and/or motor impairments. Prior to testing, subjects were given a training session to acquaint them with the nature of the stimulus and familiarize them with the testing apparatus. Subjects were trained to detect the stimulus on the tongue at a frequency of $250 \mathrm{~Hz}$ using separate descending and ascending psychophysical procedures. Standardized written instructions were routinely read to each subject during each phase of the training session. A training criterion requiring each subject to produce three threshold responses in which no two varied by more than $10 \mathrm{mV}$ was used as an index of adequate training. Subsequent to training, subjects were rescheduled for test and retest threshold assessments which occurred within a period of 10 days.

Standardized instructions were again read to each subject during each experimental session. Each subject was comfortably seated in an adjustable chair and was asked to place and hold the upper surface of the tongue against the free surround. The contactor was lowered through the hole in the free-surround disk to the anterior dorsal surface of the tongue. A negative lead from an ohmmeter was attached to the vibrator and a positive lead was held between the subject's thumb and forefinger. When the contactor touched the tongue, a complete circuit caused the 
Table 1

Means and Standard Deviations for Lingual Vibrotactile Thresholds Expressed in Microns of Peak Displacement

\begin{tabular}{llrrrrr}
\hline & & \multicolumn{5}{c}{ Frequency } \\
\cline { 3 - 7 } Condition & & 60 & 100 & 150 & 200 & 250 \\
\hline \multirow{2}{*}{ Test } & Mean & 2.6 & 1.6 & 1.9 & 1.5 & 1.5 \\
& SD & 1.2 & .7 & 1.0 & .8 & .8 \\
\multirow{2}{*}{ Retest } & Mean & 2.4 & 1.7 & 1.9 & 1.7 & 1.3 \\
& SD & 1.3 & 1.0 & 1.1 & 1.1 & .8 \\
\hline
\end{tabular}

ohmmeter to register a deflection. The static displacement gauge was adjusted to zero, and the contactor was further lowered $1 \mathrm{~mm}$ into the tongue. This procedure was used to provide a constant static contactor displacement for all subjects.

Lingual vibrotactile thresholds were obtained from each subject for test and retest sessions at the following frequencies: $60,100,150,200$, and $250 \mathrm{~Hz}$. Frequency presentations were randomized to offset possible order of presentation effects. Testing utilized an automated fixed-frequency procedure in which subjects tracked the stimulus for three successive thresholds per frequency. The median value in millivolts was accepted as threshold. Thresholds were initially recorded in millivolts and subsequently converted to microns of peak displacement using an acceleration formula.

\section{RESULTS AND DISCUSSION}

Table 1 presents a summary of the means and standard deviation for test and retest thresholds. Mean values were remarkably consistent between conditions for frequencies from 60 to $250 \mathrm{~Hz}$. The standard deviations throughout this range of frequencies were also similar and not indicative of abnormal variability. Clearly, an essentially flat response is observed for both test and retest conditions.

The flat curve across frequency is strikingly different from U-shaped functions previously observed in our research. This response is not indicative of frequencysensitive mechanoreceptors and supports the duplex theory of mechanoreception advocated by Verrillo (1968). It seems reasonable to assume that previously employed clamping procedures have distorted lingual tissues so as to excite more ventrally based energy transducers. Further testing extending the frequency range to around $500 \mathrm{~Hz}$ would provide stronger confirmation of this finding.

It would be of further interest to obtain lingual vibrotactile thresholds using a nonclamping procedure with and without the free-surround disk. Early work by Sherrick (1953), who did not clamp the tongue, showed a frequency function. Sherrick did not use a free-s surround to limit stimulus spread to more ventral lingual areas. Verrillo has contended that this was the reason for the U-shaped function Sherrick found. Further research might shed new light on this standing controversy and can provide data concerning the function of the free surround.

From a clinical perspective, these findings encourage further use of the nonclamping procedure. Since frequency does not appear to be a comparative variable for assessing surface sensitivity, it may be feasible to select an optimal frequency at which to perform screening procedures. Based on the data presented in Table $1,250 \mathrm{~Hz}$ appears to be a good choice.

Clearly, the nonclamping method considerably facilitates the testing process. A next step is to formalize training and testing procedures that work with children. Normative data, possibly at $250 \mathrm{~Hz}$, is a further requirement, as current norms were obtained using the clamping technique.

Continued clinical study of the tactile sensory system of the tongue is necessary. The clinical effectiveness of this methodology will be demonstrated in its subsequent ability to validly and reliably diagnose speech disorders that are manifested by oral sensory disturbance. The modified testing method described is another step in providing a present and future clinical tool.

\section{REFERENCES}

FuccI, D. J. Oral vibrotactile sensation: An evaluation of normal and defective speakers. Journal of Speech and Hearing Research, 1972, 15, 179-184.

Fucci, D., \& Kelly, D. New instrumentation for research on vibrotactile sensitivity of the tongue. Review of Scientific Instruments, 1972, 43, 1748-1751.

Geldard, F. A. The human senses (2nd ed.). New York: Wiley, 1972.

SHERRICK, E. C., JR. Variables affecting sensitivity of the human skin to mechanical vibration. Journal of Experimental Psychology, 1953, 45, 273-282.

Telage, K., \& FuCCI, D. Vibrotactile stimulation: A future clinical tool for speech pathologists. Journal of Speech and Hearing Disorders, 1973, 38, 442-447.

Telage, K., \& Fucci, D. Concerning intrasubject measurements of successive lingual vibrotactile responses. Perceptual and Motor Skills, 1974, 39, 1047-1052. (a)

Telage, K., \& Fucci, D. Measurement of lingual vibrotactile sensitivity using one-trial and three-trial threshold criteria. Bulletin of the Psychonomic Society, 1974, 3, 373-374. (b)

Telage, K., \& Fucci, D. The effects of linear and logarithmic data transformation upon observed patterns of lingual vibrotactile sensitivity. Bulletin of the Psychonomic Society, 1975, 6, 210-212.

Verrillo, R. T. Specificity of a cutaneous receptor. Perception \& Psychophysics, 1966, 1, 149-153.

VerRILlo, R. T. A duplex theory of mechanoreception. In D. Kenshalo (Ed.), The skin senses. Springfield: Charles C Thomas, 1968. Pp. 139-156.

Winkelman, R. K. Nerve endings in normal and pathologic skins. Springfield: Charles C Thomas, 1960.

(Received for publication June 19, 1978.) 